\title{
WEED AND CROP SEGMENTATION AND CLASSIFICATION USING AREA THRESHOLDING
}

\author{
Su Hnin Hlaing ${ }^{1}$, Aung Soe Khaing ${ }^{2}$ \\ ${ }^{1}$ Demonstrator, Department of Electronic Engineering, Mandalay Technological University, Myanmar \\ ${ }^{2}$ Associate Professor, Department of Electronic Engineering, Mandalay Technological University, Myanmar
}

\begin{abstract}
In the agricultural industry, the weed and crop identification and classification are major technical and economical importance. Two classification algorithms are focused in this paper. And the better classification algorithm has been selected to classify weed and crop from the images. There are three main parts of proposed system are segmentation, classification and error calculation. The developed algorithm based on area thresholding has been tested on weeds and various locations. Forty one sample images have been tested and the result of some weed coverage rate is illustrated. Moreover, the misclassification rate is also computed. An algorithm has been done to automate the tasks of segmentation and classification. The overall process is implemented in MATLAB.
\end{abstract}

Keywords - Objects segmentation, Image processing, Plant classification, Area Thresholding

\section{INTRODUCTION}

As weeds are frequently distributed non -uniformly within a field, weeding is a very hard work [1]. Every year a large amount of herbicide is used for removing weeds from agricultural fields which is not only expensive but also a source of environmental pollution. Moreover, both costly and time consuming is caused due to hand labor [4]. Therefore, weed control is a necessary management practice in agricultural systems, which is critical to sustain crop productivity and quality [1]. Farmers need alternatives for weed control due to the desire to reduce chemical use and production costs [7]. For instance when growing vegetables such as carrots, onions and cabbage the weeds can be removed by special harrows but it cannot support for economic system [3].

Nowadays, uniform spraying is the most common method for herbicides application. However, this method is inefficient and cost-ineffective as weed distribution is usually non-uniform and highly aggregated in clumps within the arable field [1]. There could be many parts of the field that have none or insignificant volume of weeds. This property of the weed distributions makes the development of site-specific management feasible [8]. In this management, the amount of herbicides applied is reduced through spraying only the weed infested area of a field, where different selective herbicides with corresponding application rates are applied to control weed differently [5]. The machine vision based approach uses shape, texture, color and location based features individually of jointly to discriminate between weed and crop [11]. In the other research, Caltrans sprays roadside plant material with herbicide to prevent the weeds from becoming a fire hazard during the summer. The first step in identifying weeds within an image involves classifying the pixels [12]. The pixels shall be classified using a point operation. The surrounding pixels will not bias a pixel's classification. The purpose of segmenting the image into plant and background pixels is to detect the amount of plant material within a specific area [12]. If the amount of plant material reaches a specific threshold, the area is targeted for herbicidal spray application [12]. The spray threshold is set too close to the background misclassification rate, then herbicide will be wasted spraying background. Therefore, a larger misclassification rate limits the smallest plant that can be detected without targeting the background for spray [12].

A system that could make use of the spatial distribution information in real-time and apply only the necessary amounts of herbicide to the weed-infested area would be much more efficient and minimize environmental damage. Therefore, a high spatial resolution, real-time weed infestation detection system seems to be the solution for site-specific weed management.

In this paper, three main parts presented for weed and crop classification. It has got (1) Image acquisition and gray 
transformation, (2) Image segmentation and noise removal, and (3) Classification of weed and crop [2]. Two classification methods are studied in this research. They are classification based on intensity method and area thresholding classification method. The first method is depended on the intensity of the images [13]. However, this algorithm cannot operate to classify weed and crop automatically. So, the area thresholding method is used to get reliable classified images. These methods are executed depending on size of the plants. By using digital camera, the input image is obtained. Most of images are needed to change from the color images into grayscale images for easy and fast processing. The segmentation step implemented by using thresholding method. In the classification part, the areas of segmented image are then compared with the selected threshold for classification of weeds and crop [6].

\section{MATERIALS AND METHODS}

The first algorithm consists of five fractions: (i) preprocessing, (ii) Binarization using Otsu's thresholding, (iii) Marker control watershed segmentation, (iv) Gray transformation and (v) classification based on intensity. The simple weed and crop images are used to test the system. Firstly, the color images are converted to gray scale images for easy and fast processing [13]. Median filter is applied to the gray image to reduce the amount of data. Otsu's method is used to filtered image for converting black and white image. This method chooses the optimal threshold to minimize the intraclass variance of the black and white pixels [14]. Then, binarized image is divided into different regions according to watershed segmentation method. The sobel operator is applied on the binary image to find the gradient magnitude. By using this magnitude, estimate the watershed transform that divide different regions. The segmented image is changed into RGB segmented image to distinct region. To classify weed and crop, the RGB image is converted into gray image. Finally, the intensity value is defined by manually to extract weed plant from the image. The results image is described in Fig- 3. The intensity values of the images can change due to light, dark, weather condition and camera situation. Therefore, these values must be defined for every weed and crop images. However, the next algorithm can detect without manual extraction as can be seen their results image.

The area thresholding classification system includes the five steps. They are Excess Green Gray transformation, segmentation, label the image, removing the unwanted data and classification based on area thresholding. The system block diagram of the system is illustrated in Fig-1, which consists of three main steps:

1) The Excess green gray transformation $2 \mathrm{G}-\mathrm{R}-\mathrm{B}$ is executed to easy and fast processing for segmentation stage.
2) Background and plants of the image is separated according to the gray index.

3) Extraction of weed and crop from the segmented image by area thresholding.

And then the detailed algorithms of the system are also described into step by step.

\subsection{Image Acquisition}

In this research, the weed and crop color images are acquired through the digital camera. The sample images are captured in the fields. Images are obtained at different times of a day. Moreover, weeds and crop with varying canopy size were selected to increase the difficulty of the classification problem. The weed and crop images are taken at an angle 45 degree with the ground in natural lighting conditions with digital camera. The camera is mounted in the height of $2.15 \mathrm{~m}$ from the ground. The resolution of the camera is set to $3648 \times 2736$ pixels during image capturing. In the experimental analysis, all the images were resized to a resolution of $320 \times 240$ pixels in order to reduce the computation time [10]. Fig-2 illustrates sample weed and crop images taken from the fields.

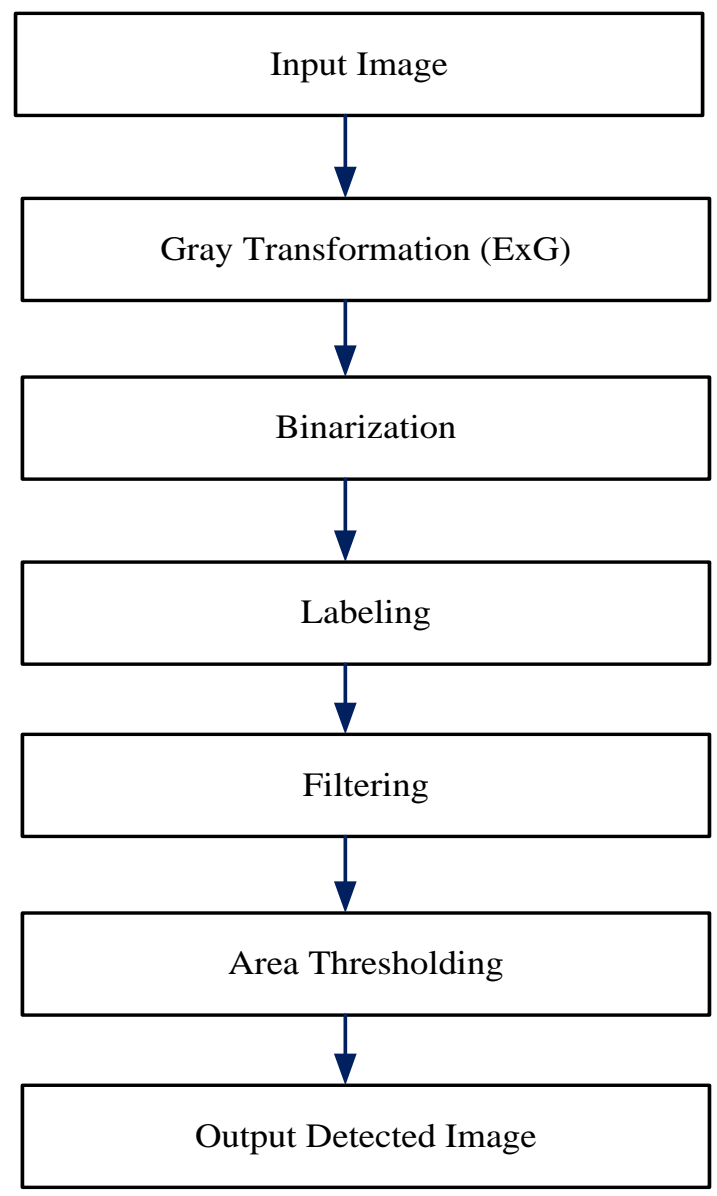

Fig-1: Block diagram of the system 


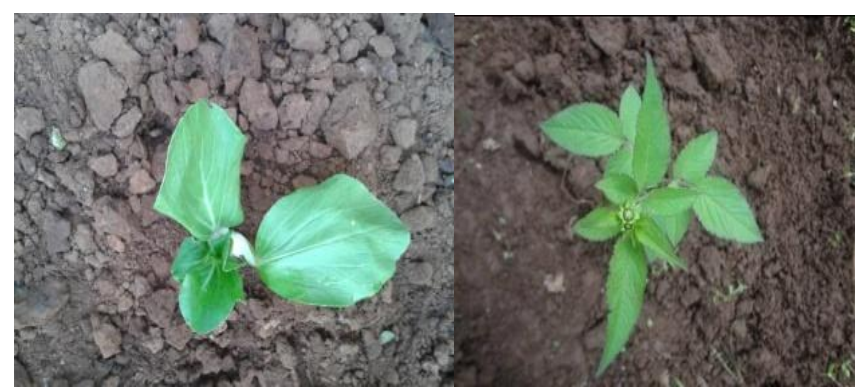

(a)

(b)

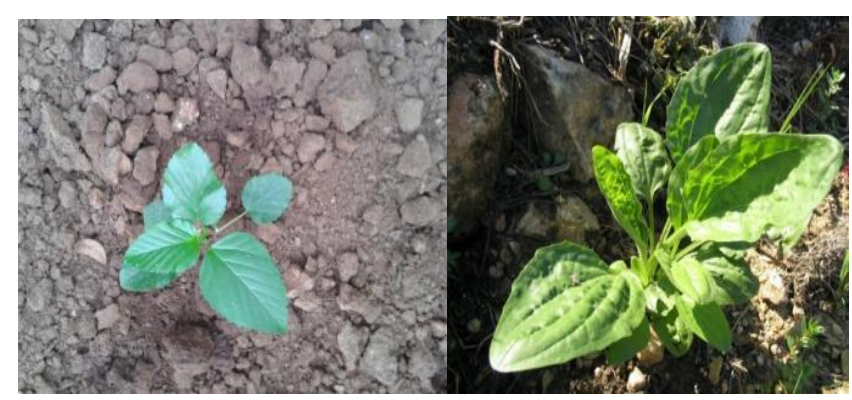

(c)

(d)

Fig-2: The original images of the crop and some weed that captured by using camera: (a) rape plant, (b) Lanchon, (c) Pigweed and (d) Kyaut kut.
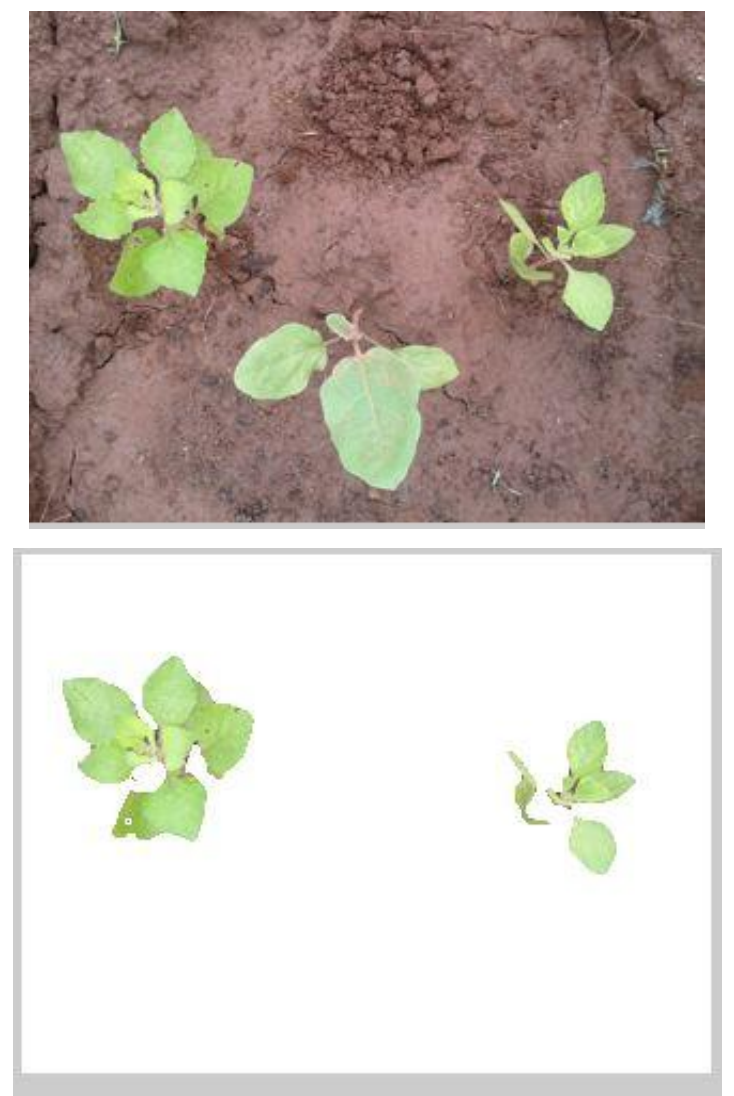

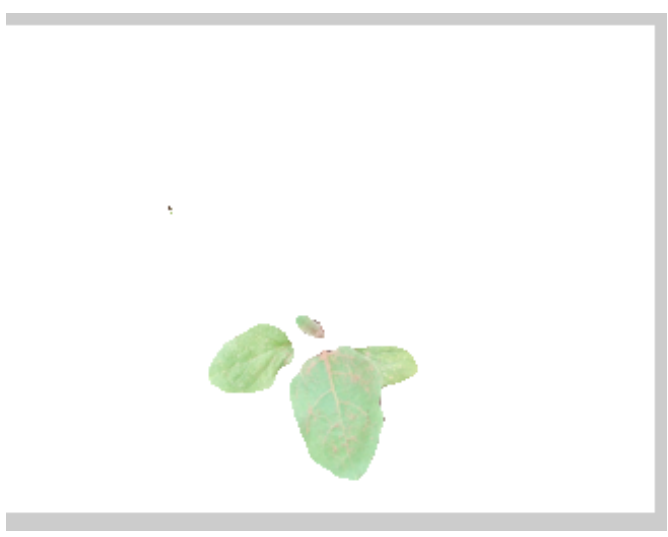

Fig-3: Sample result of classification based on intensity method: Original image (up); Detected weed image (bottomleft); Detected crop image (bottom-right).

\subsection{Gray Transformation}

The gray transformation processing is to turn the color image to the gray image. The purpose of gray transformation is to reduce the amount of color data in the image so as to speed up the following processing. The color difference between plants and background in the color images should be kept as well as possible in the gray image. Equation (1) is used for gray transformation in image processing. Assuming the coordinate $(x, y)$ is the pixel point.

$$
\begin{gathered}
\operatorname{EXG}(\mathrm{x}, \mathrm{y})=2 \mathrm{~g}-\mathrm{r}-\mathrm{b} \\
\text { Where, } \mathrm{r}=\frac{R}{\mathrm{R}+G+B}, \mathrm{~g}=\frac{G}{R+G+E}, \quad \mathrm{~b}=\frac{B}{R+G+B}
\end{gathered}
$$

In this equation $\mathrm{R}, \mathrm{G}$ and $\mathrm{B}$ are the three components of pixel color in RGB color space; ExG is the transformation result, a gray value. The intensity information is highlighted and discarded most color information in the color image using equation (1). The pixel values in red channel and blue channel color space are always less than the in green color. Therefore, the Excess green feature is used to extract the distinct color channel. The result of this algorithm is illustrated in Fig- 4.

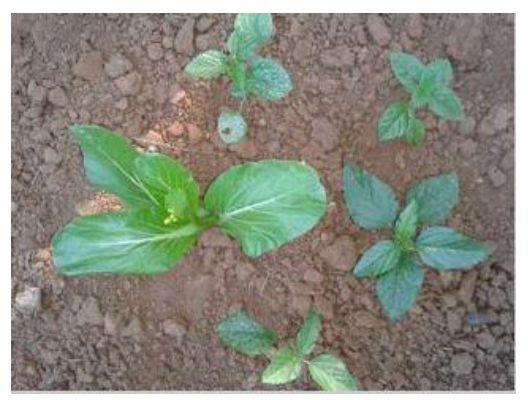

(a) The original input image 


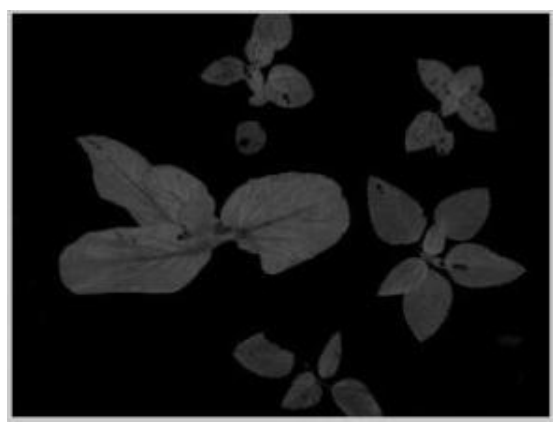

(b) Result of the Green minus red image

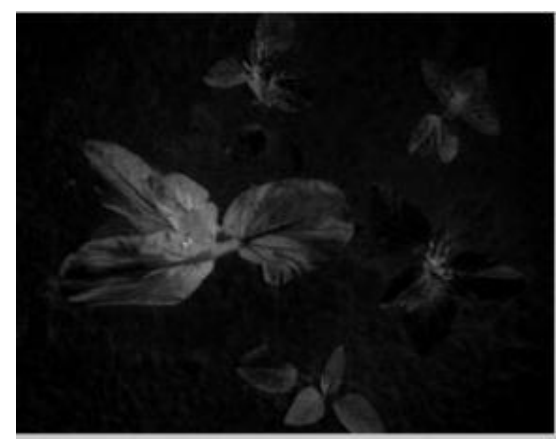

(c) Result of the green minus blue image

Fig-4: Gray transformation

\subsection{Segmentation}

To remove background of the image, different techniques such as thresholding-based segmentation, edge-based segmentation, Color-based segmentation and watershed segmentation can be used. In this study, gray removal segmentation method is used for the segmentation task. All the segmentation steps are done depending on the binary image. Therefore, the grayscale image is converted into binary image from the preprocessing stage. Thresholding based on gray image is used to change the binary image. By using the following equation

$$
g(x, y)=\left\{\begin{array}{lr}
P, & |G-R|>T \text { and }|G-B|>T \\
B g, & \text { Otherwise }
\end{array}\right.
$$

For all pixels in the original image the absolute values of green minus red and green minus blue are calculated. These give measurements of the pixels distance to the grayscale line. If both of these distance values are greater than threshold $(\mathrm{T})$, the pixel is classified as plant $(\mathrm{P})$. If none or only one is greater than $\mathrm{T}$, the pixel is classified as background $(\mathrm{Bg})$. The number of $\mathrm{T}$ is equivalent to the threshold in the above mentioned index methods and has to be chosen based on the available image material. For the image of this paper $\mathrm{T}=20$ has proven to give a very good result. This method of removing pixels of high 'greyness' can be seen as calculating two separate indices and then requiring that a pixel is on the correct side of the threshold in both cases to be classified as $\mathrm{P}$. The result of binary images includes the small noise as misclassified plant pixels. Therefore each pixel of the segmented images is labeled with a value according to the component it is assigned to. After that the property of the region is defined into area. Then the value of region is firmed to find the minimum area. And then the minimum area of the region is removed from the image as shown in fig-5. The 40 pixels of minimum areas are discarded in this filtering stage.

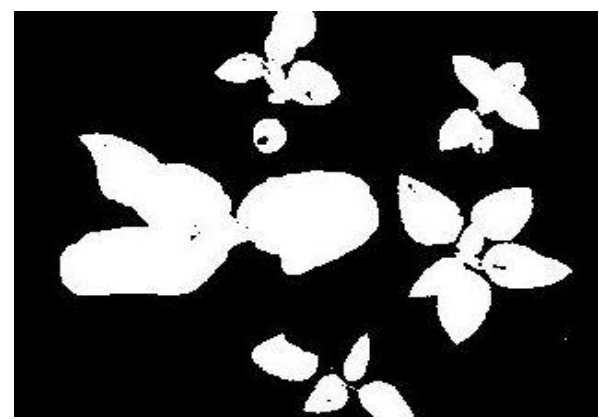

(a)

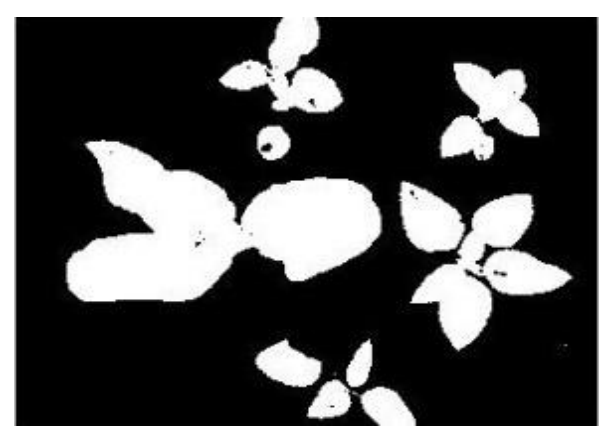

(b)

Fig-5: Result of segmented (a) binary image and (b) filtered image

\subsection{Classification}

The final segmented image is used as the input of the weed and crop classification stage. Weed detection is executed depended on their areas. Firstly, the segmented binary image is labeled using 8-connected components. In this way, it can easy to evaluate the areas of the objects. And then, a threshold value is selected to classify weed plants and crop plants. Therefore, threshold value is set at 6000 for this research. The crop plants are mostly large the weed plants in related to the testing. Therefore, the weed plant is detected when the object areas are less than threshold value. In the other way, the remaining objects are classified as crop plants. The areas of the individual objects are calculated according to the equation (3). In this equation, $F(j, k)$ is binary segmented image, $x_{j}$ and $y_{k}$ are scaled coordinates in row and column and $\mathrm{J}$ and $\mathrm{K}$ are row and column of binary 
image. The detected weed and crop images are obtained by comparing the threshold value and their areas.

$$
M(m, n)=\frac{1}{J^{m} K^{n}} \sum_{j=1}^{J} \sum_{k=1}^{K}\left(x_{j}\right)^{m}\left(y_{k}\right)^{n} F(j, k)
$$

The desired points of the image $\left(\mathrm{x}_{\mathrm{j}}\right.$ and $\left.\mathrm{y}_{\mathrm{k}}\right)$ are obtained using the following equation:

$$
\begin{gathered}
x_{j}=j+1 / 2 \\
y_{k}=k+1 / 2
\end{gathered}
$$

The thresholding equation is illustrated in equation (4). The optimal threshold value is selected at 6000 .

$$
I= \begin{cases}\text { weed, } & \text { area }<T \\ \text { crop, } & \text { area } \geq T\end{cases}
$$

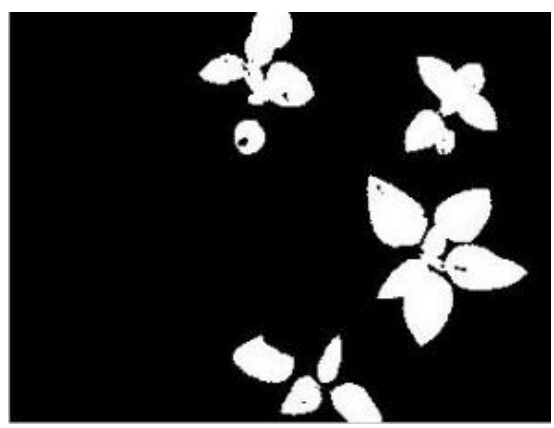

(a) Result of detected weed image

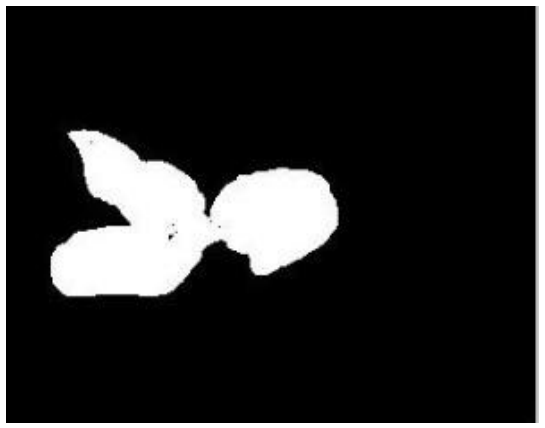

(b) Result of detected crop image

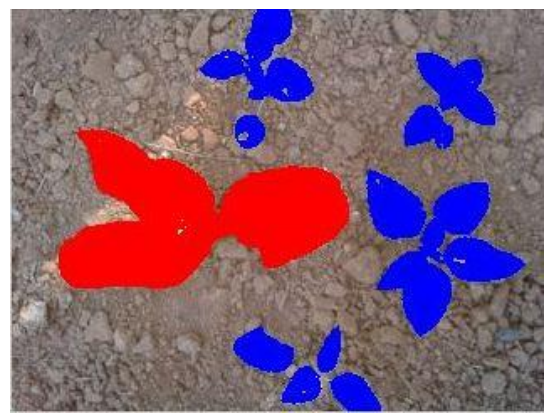

(d) Result of classified image

Fig-6: Result of weed and crop classified images
If the sizes of the crop plants are equal to the weed plants, the classification algorithm can cause the misclassification. Therefore, those plants are misclassified results as can be seen in Fig. 6. This original image consists of two crop plants and four weeds plants. Among the two crop plants, the area of the one crop plant is less than the system threshold value. So, the misclassified image is obtained as can be seen the Fig-7. (b).

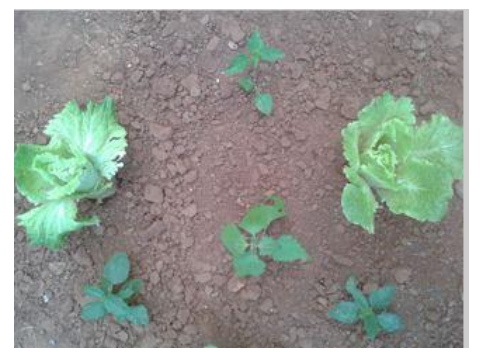

(a) Original weed and crop image

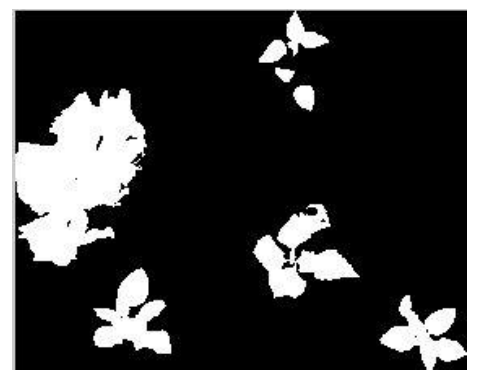

(b) Detected weeds image with one crop is misclassified as weed

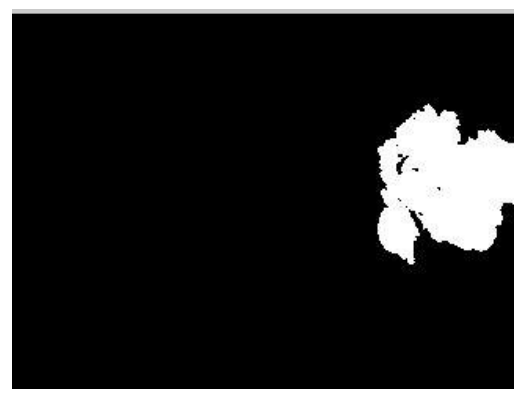

(c) Detected crop image

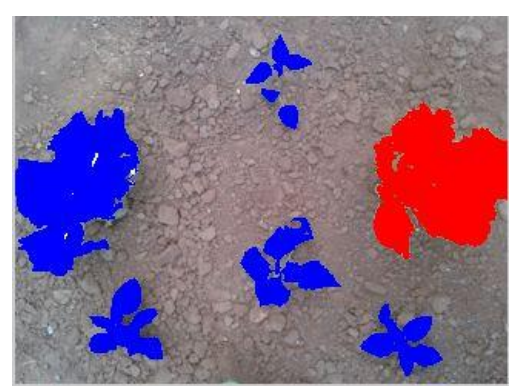

(d) Misclassified image

Fig-7: Result of misclassified weed and crop images. 
Table- 1: The Experimental Results of Area Thresholding

\begin{tabular}{ccccc} 
Num & $\begin{array}{c}\text { Total } \\
\text { Area }\end{array}$ & $\begin{array}{l}\text { Weed } \\
\text { Area }\end{array}$ & $\begin{array}{l}\text { Plants } \\
\text { Coverage }\end{array}$ & $\begin{array}{l}\text { Detected } \\
\text { weed } \\
\text { Coverage }\end{array}$ \\
\hline 1 & 21986 & 6574 & $28.60 \%$ & $9.19 \%$ \\
2 & 9029 & 17260 & $22.50 \%$ & $12.60 \%$ \\
3 & 9069 & 17406 & $22.70 \%$ & $12.60 \%$ \\
4 & 10985 & 2891 & $14.30 \%$ & $3.80 \%$ \\
\hline
\end{tabular}

In the Table1, plants coverage and detected weed coverage rate are defined as:

$$
\begin{gathered}
\text { Plants Coverage }=\frac{\text { Total Area }}{\text { Zone Area }} \times 100 \% \\
D=\frac{\text { sum } w}{\text { Zone Area }} \times 100 \%
\end{gathered}
$$

In Table 1, data describes that the percent of the plants coverage and detected weed coverage of the results images. According to the above equations SUMw is the sum of all weed pixels and Zone Area is the pixel count in the whole image, and is equal to the row $\times$ column of the image pixels. As a result, almost of $13 \%$ of weed coverage is found in the sample images.

$$
\mathrm{e}_{\mathrm{r}}=\frac{\mathrm{Nm}}{\mathrm{Nw}} \times 100 \%
$$

Where, $e_{\mathrm{r}}=$ error rate or misclassification rate

$\mathrm{Nw}=$ number of detected weeds

$\mathrm{Nm}=$ number of misclassified crop

Above the error equation (7) is used to compute the misclassification rate. As the sample results is illustrated in Table II. For example, the first image in this table has six weed plants and three crop plants. But the number of output detected weed plants are nine. So, all the crop plants are defined as weed plants. Therefore, $33.3 \%$ error rate is obtained when these misclassified plants is evaluated according to the equation (7). The remaining error rate can be generated in the same way.

\section{RESULTS AND DISCUSSION}

This paper presents two main algorithms for weed and crop classification system. Fig- 6 and 7 describe the classified result images. The plants with blue color are defined as weeds and the red plants are classified as crops. The given algorithm processes two types of images for weed and crop. The algorithm produced reliable classified images to be sprayed. In the field, almost the images that captured according to image acquisition step can be classified to give to the spray system. By using the resultant image, the weed position can determined according to the pixels of left to right and top to bottom. Although the system could classify weed to be sprayed, darker images cause more errors in the segmentation and also in later steps of the algorithm. When the weed and crop plants are overlapped, this method cannot classify weed and crop. The 41 sample images have been used to test in this study. Among them, 7 images are found the misclassification. Thus the proposed algorithm is suitable for weed and crop segmentation and classification. It will support to get the reliable results in real-time application.

Table-2: Error Calculations of misclassified Weed and Crop Images for the Area Thresholding Algorithm

\begin{tabular}{ccccc}
\hline No. & $\begin{array}{c}\text { Number } \\
\text { of } \\
\text { detected } \\
\text { weeds }\end{array}$ & $\begin{array}{c}\text { Number of } \\
\text { misclassified } \\
\text { crop }\end{array}$ & $\begin{array}{c}\text { Missed } \\
\text { weeds }\end{array}$ & $\begin{array}{c}\text { Weed mis- } \\
\text { classifications } \\
\text { rate }\end{array}$ \\
\hline 1 & 9 & 3 & 0 & $33.30 \%$ \\
2 & 6 & 1 & 0 & $17 \%$ \\
3 & 5 & 1 & 0 & $20 \%$ \\
4 & 4 & 1 & 0 & $25 \%$ \\
5 & 5 & 0 & 0 & $0 \%$ \\
\hline
\end{tabular}

In this Table 2, error calculations have been executed by manually counting the number of misclassifications in the randomly chosen result images. The missed weeds in this Table II are weeds that were not fully marked in red or blue. The weed misclassifications shows the objects classified as weeds that are actually part of crop plant. Both weed area is greater than threshold value and crop area is less than threshold value can cause the misdetection. Although the second system has some drawback, the better classification results could generated with small error.

\section{CONCLUSIONS}

In this research, segmentation method and classification based on area thresholding method are developed. Excess green gray transformation (ExG) and area thresholding algorithms are combined to obtain the exactly classified images. The system shows an effective and reliable classification of images captured by a camera. The image segmentation algorithm is very useful method in the image processing and it is very helpful for the subsequent processing. When the plants are separated from each other in the images, the results have been shown to be better. Also the lighting conditions are important to be able to make a reliable analysis. 


\section{ACKNOWLEDGMENT}

The authors wish to thank to the head of Department of Electronic Engineering, Mandalay Technological University. The author would like to express special appreciation and heartfelt thanks to her supervisor, Dr. Aung Soe Khaing, Department of Electronic Engineering, Mandalay Technological University for giving understanding, helpful guidance, suggestion and directions throughout the preparation of work. The author is also sincerely thankful to all her teachers, Department of Electronic Engineering, Mandalay Technological University.

\section{REFERENCES}

[1]. Faisal Ahmed, Hasanul Kabir, Shayla Azad Bhuyan, Hossain Bari, and Eman Hossain, "Automated Weed Classification with Local Pattern-Based Texture Descriptors", International Arab Journal of Information Technology, vol.9, no.4, pp. 382-391.

[2]. Irshad Ahmad, Abdul Muhamin, Muhammad.Islam and Shahid Nawaz,"Weed Classification using Histogram Maxima with Threshold for Selective Herbicide Applications", World Academy of science, Engineering and Technology 2007.

[3]. Siddiqi M., Sulaiman S., Faye I., and Ahmad I., "A Real Time Specific Weed Discrimination System Using Multi-level Wavelet Decomposition", International Journal of Agriculture and Biology, vol.11, issue 5, pp.559-565,2009.

[4]. Hossein Nejati, Zohreh Azimifar and Mohsen Zamani,"Using Fast Fourier Transform for Weed Detection in Corn Fields", IEEE International Conference on Systems, Man and Cybernetics (SMC 2008).

[5]. Mahammad Hameed Siddiqi, Sungyoung. Lee, YoungKoo Lee, "Efficient Algorithm for Real-Time Specific Weed leaf Classification System", Journal of Communication and Computer, ISSN 1548-7709, USA.

[6]. S.Kiani and A.Jafari, "Crop Detection and Positioning in the field Using Discriminant Analysis and Neural Networks Based on shape features", J-Agr.Sci.Tech (2012) Vol.14:755-765.

[7]. Sajad KIANI, "Discriminating the Corn Plants from the Weeds By Using Artificial Neural Networks", International Joural of Natural and Engineering Sciences 6 (3): 55-58, 2012 ISSN: 1307-1149,E-ISSN: 2146-0086, WWW.nobel.gen.tr.

[8]. Tian L., Reid J., and Gerhards R., "Development of a Precision Sprayer for Site-specific Weed Management", Transactions of the ASAE, vol.42, issue 4, pp.893-900, 1999.

[9] DIAO ZHIHUA, WANG HUAN, SONG YINMAO, and WANG YUNPENG, "Image Segmentation
Method for Cotton Mite Disease Based on Color Features and Area Thresholding", Journal of Theoretical and Applied Information Technology, $10^{\text {th }}$ February 2013.Vol. 48 No.1, ISSN: 1992-8645.

[10]. W.S. LEE, D. C. SLAUGHTER, AND D.K. GILES, "Robotic Weed Control System for Tomatoes", Precision Agriculture, 1, 95-113(1999)

[11]. Anant Bhardwaj, Manpreet Kaur, and Anupam Kumar, "Recognition of Plants by Leaf Image using Moment Invariant and Texture Analysis", International Journal of Innovation and Applied Studies ISSN 2028-9324 Vol.3 No.1 May 2013, pp.237-248, http://www.issrjournals.org/ijias/

[12]. Chris Gliever, EEC206 Project Report, "Color Segmentation of Plant and soil". 3177 EUII, Friday 5PM, March $21^{\text {th }} 2003$.

[13]. Irshad Ahmad, Muhammad, Iram Fatima, Sungyoung Lee, Young-Koo Lee, "Weed Classification Based on Haar Wavelet Transform via k-Nearest Neighbor (k$\mathrm{NN)}$ for Real-Time Automatic Sprayer Control System", ICUMC 11, February 21-23,2011, Seoul, Korea.

[14]. Mehmet Sezgin, Bulent Sankur. "Survey over Image Thresholding Techniques and Quantitative Performance Evaluation", Journal of Electronic Imaging. 13 (1): 146-165, 2004.

\section{BIOGRAPHIES}

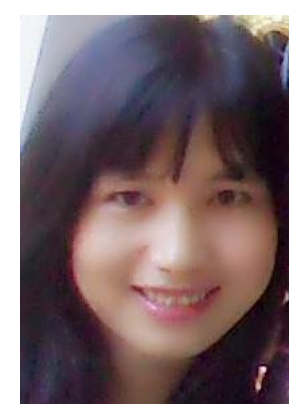

Su Hnin Hlaing received her Bachelor of Engineering in Electronic Communication Technology from the Mandalay Technological University, Myanmar in 2008. She is now master student in the Mandalay Technological University, Myanmar. Her research interests include image processing, weed and crop segmentation and classification.

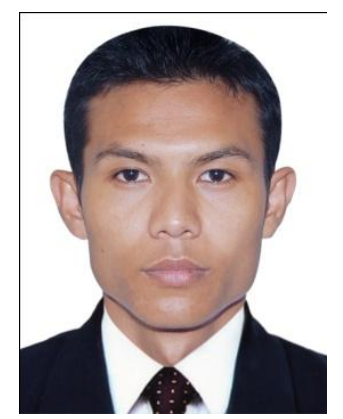

Aung Soe Khaing received his $\mathrm{PhD}$ in Electronic Engineering from Mandalay Technological University, Mandalay, Myanmar, in 2011. He was doing research on Spatial Frequency Analysis of the Human Brain at the Institute of Biomedical Engineering and Informatics, Technical University Ilmenau, Germany. $\mathrm{He}$ is now Associate Professor at 


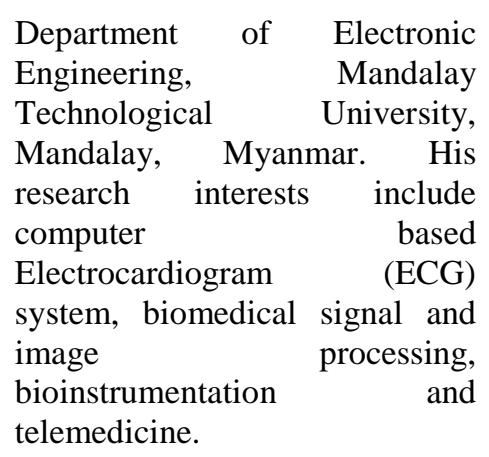

